\title{
Arbor
}

\section{La mujer, crítico de arte}

Rosa Martínez de Lahidalga

Arbor CLXVIII, 663 (Marzo 2001), 309-319 pp.

La labor desempeñada por la mujer en el ejercicio de la crítica de arte en el último tercio del siglo es notoria. Autora de libros, artículos y ensayos que ven la luz en diarios y en revistas especializadas, participa en jurados nacionales e internacionales, comisaría de exposiciones y con frecuencia interviene como conferenciante. Enseñar a mirar la obra de arte es tarea primordial que mueve a su actividad, en un intento no solo de informar sino de aproximar la obra de arte al contemplador y hacer que sea cada vez más estrecha la línea divisoria entre arte y público.

Del 10 al 17 del pasado mes de septiembre se celebró en Londres, en la sede de la Tate Modern, el Congreso anual de la Asociación Internacional de Críticos de Arte. En la organización y desarrollo del mismo participaron, junto con personal especializado de la Tate, que ha recuperado para su espacio lo que fue una central eléctrica anclada junto al Támesis, representantes del Departamento de Artes Visuales del Goldsmiths College.

Más de trescientos profesionales debatieron desde puntos de vista diversos, en torno al que fue tema central del mismo: Arte Visual o Cultura Visual. La reunión puso de manifiesto, no solo la diversidad de criterios de los participantes, sino también la heterogeneidad de profesionales que hoy tienen cabida en la Asociación, además de críticos de arte, galeristas, directores de museo, profesores, curators o comisarios de exposiciones, gestores de colecciones privadas o públicas e incluso artistas. De la totalidad de asistentes, casi el ochenta por ciento eran mujeres procedentes de los siguientes países: Australia, Austria, Barbados, Bulgaria, 
Croacia, Estados Unidos, Hungría, Italia, Jamaica, Macedonia, Nigeria, Polonia, Reino Unido, República Dominicana, Rusia, Suiza y España. Cuarenta de ellas presentaron ponencias o participaron en el turno de intervenciones anunciadas, en tanto que la participación masculina se redujo en número a la mitad.

La presencia de la mujer en el área cultural es un hecho que deja en el pasado las llamadas de atención que a principios de siglo lanzaran hombres de la categoría de Américo Castro, al manifestar abiertamente que «la vida española necesita de la mujer, si es que aspira a soltar su dejo de rusticidad»; de Ortega y Gasset, quien reclamó su asistencia a las aulas, o de Eugenio d'Ors, favoreciendo su inserción en el terreno de las artes. Tendría que transcurrir hasta el último tercio del siglo para que la incorporación de la mujer a la práctica totalidad de las áreas del saber fuera una realidad.

Su actividad se hace sentir tímidamente desde principio del siglo en el terreno de la enseñanza, de la literatura y del pensamiento. También lo hace en el de la critica de arte, si bien su participación es todavía más minoritaria. En 1905 Emilia Pardo Bazán (1852-1921) escribe su novela La Quimera, en la que el principal protagonista es un pintor. Bajo el nombre de Silvio Lago, se oculta la personalidad del artista coruñés Joaquín Vaamonde (1872-1900), artista malogrado que había realizado un excelente retrato de la condesa en 1896. Aun cuando no se trata de crítica de arte en sí, resulta significativo que sea una mujer la que por vez primera incluya en su mundo literario el personaje de un pintor, y que además emita juicios realmente acertados sobre el ambiente artístico y sobre la psicología del artista.

Hacia 1912 surgía a la crítica de arte la escritora Margarita Nelken. Casada con el también crítico García Maroto, que publicaba sus crónicas en El año artístico, Margarita Nelken publica en 1917 su libro Glosario (Obra y artistas). A propósito del mismo, Juan Antonio Gaya Nuño en su libro, Historia de la crítica de arte en España ${ }^{1}$ expresa su sorpresa ante la valentía y novedad de sus juicios y el conocimiento que demuestra tanto sobre el arte español del momento como sobre el que se realiza en el extranjero.

En los años 20 la escritora hacía la crítica de arte en El Figaro. En una de sus crónicas, a la vista del grabado de Picasso, La comida frugal, conocido también como El viejo y su compañera, comenta: «Es la obra de Picasso y es la obra nuestra, la que nos corresponde, la que nos satisface con sencillez, que encierra todas nuestras complejidades. Y hay algo en su pureza que está más alto que todos los trabajos, que lo aísla de los trabajos hechos para el arte y que lo hace estáticamente, solo para la vida». 
El juicio contrasta por su agudeza, con el que emite por entonces sobre el pintor malagueño, el crítico Ricardo Gutiérrez Abascal, que firma con el seudónimo de Juan de la Encina: «Para mi Pablo Picasso es pintor de gran talento; pero en ningún caso un precursor de tiempos nuevos, un abridor de verdaderos caminos. Más bien que un iniciador, es un epígono en rapsoda. Discurrir a lo largo de su obra es parecido a recorrer un poco precipitadamente por todo el arte moderno y no poco del antiguo. Picasso lo sabe todo y todo lo imita y reproduce». Gaya recoge en su libro estas palabras y añade: «lastimosa la exencia de olfato, a comparar con el muy fino de que había dado pruebas Margarita Nelken siete años antes» ${ }^{2}$.

El ejercicio de la crítica de arte como actividad, no sólo informativa, sino valorativa y dinamizadora, era por entonces prácticamente inexistente. En estas circunstancias, uno de los acontecimientos más importantes para el arte español lo constituyó la fundación de la Academia Breve de Críticos de Arte. Solo por este hecho, de la total autoría de Eugenio d'Ors, merecería figurar su nombre en la nómina de los conductores y orientadores de la cultura artística del siglo. El escritor y crítico de arte Enrique Azcoaga, primer Secretario que tuvo la Academia, había conocido a d'Ors en mayo de 1940 y en carta escrita desde su residencia en México, a Manuel Sánchez Camargo, recordaba: «La idea inicial de d’Ors consistía en aprovechar el prestigio de los colaboradores reunidos entonces en la revista Santo y Seña, en un homenaje a El Greco, para defender el arte moderno. En España decir arte moderno era ofender a toda una serie de lázaros ramplones y anacrónicos enemigos hasta la nausea de la auténtica vanguardia». ${ }^{3}$

En el momento en que nace la Academia Breve la formación académica del artista era sólida y el dominio del oficio bueno, pero el resultado de la obra era frío e incluso amanerado. El respeto a la tradición sirvió de base al magisterio de d' Ors, que delimitó fronteras estéticas y enseñó a través de sus glosas y de sus libros, entre ellos Mis Salones, Cézanne o los dedicados al Museo del Prado, hasta que punto era necesario abandonar los usos de las Exposiciones nacionales.

Artistas como Regoyos, Nonell y Solana cubrían con creces la mejor línea de creatividad, pero trabajaban aislados. Fuera de nuestras fronteras triunfaban las llamadas «escuelas de París» con Picasso y Juan Gris a la cabeza. El problema afectaba en España a una juventud que experimentaba deseos innovadores y no encontraba cauces para llevar a término su obra si no era con grandes dificultades. Ejemplo de ello fue la situación de desamparo que vivieron en sus comienzos artistas como Benjamin Palencia y Godofredo Ortega Muñoz o el mismísimo Solana. 
La Academia respondió a esa necesidad. En ella hizo su primera exposición Antonio Tàpies y a su amparo se consagró Nonell públicamente como el maestro que era, cuando todavía carecía de reconocimiento. Con todo, lo más importante de la institución fue que a través de ella tomó forma una nueva conciencia entre los estudiosos y críticos de arte y el escaso público que por entonces visitaba las pocas exposiciones que se celebraban. La Academia Breve de Crítica de Arte celebró su primer acto público en mayo de 1942 en la galería Biosca. Poco antes y en reunión previa, había quedado establecida la primera cláusula, en la que se especificaba que el número de miembros sería once, tal como estaba previsto. En el acto inaugural hubo un solo cuadro de Nonell, Gitana, que fue cedido por el Dr. Blanco Soler, de su colección particular. El resto de las obras se pudieron mostrar al público el 16 de Julio, en un Madrid desierto por las vacaciones.

En el catálogo de la exposición justificaba d'Ors el nacimiento de la Academia por el interés de un grupo de hombres de cultura a los que animaba la defensa e ilustración de las corrientes artísticas modernas. Y aprovechaba para denunciar a su vez la escasa preparación artística de crítica y público, haciendo hincapié en los primeros «ayunos muchos de ellos de conocer una sola página de arte contemporáneo universal» ${ }^{4}$. No eran palabras halagadoras pero respondían a la verdad.

En el I Salón de los Once, que abrió sus puertas en el otoño de 1943 en la Galería Biosca, figuraban obras de María Blanchard y Olga Sacharoff junto a las de Zabaleta, Pedro Bueno, Eduardo Vicente, Fujita, Jesús Olasagasti, Pedro Pruna, Manolo Hugué, José Serrano y Emilio Grau Sala. De Maria Blanchard, que había fallecido en París en 1932, se mostraron trece pinturas. Era la primera vez que el público contemplaba obras de la artista, a pesar de que había vivido y pintado varios años en Madrid. Entre las personalidades que integraron el I Salón se encontraba María Laffitte, Condesa de Campo Alange, quien celebró una sesión de lectura sobre María Blanchard, de la que escribiría un libro en $1944^{5}$. En el II Salón de Otoño, Eugenio d'Ors presentó la obra de Rosario de Velasco; Enrique Azcoaga a Benjamin Palencia; la Condesa de Campo Alange, a José Serrano; José María Alfaro a Joaquín Torres García... Estos y otros datos hablan ya de una incipiente presencia femenina literaria y artística.

El mismo año 1943 se creaban Los Amigos de la Academia Breve de Crítica de Arte, dando cabida a políticos y personalidades de la vida social. Constituían, en palabras de su fundador, un «Estado Mayor que había de orientar los ejércitos de renovación del arte y de la vida artística en España». Eran 171 miembros, de los que más de treinta eran mujeres. 
Poco después establecía las bases que habían de presidir la función crítica. Consideraba como primer ejercicio de la crítica el de recapacitar. «Tras la recapacitación viene la comparación. Ese comparar y comparar; jugar términos opuestos, contrastar valores de ayer con propias aportaciones; sobre todo dialogar, abrir miradores para que todos digan con seguras razones los comos y los porqués». Y aún añadía que cumple al crítico la misión de propagar lo que se hace en arte. Después le corresponderá elegir. Esa misma misión le atribuye a la Academia Breve: «Cuando expone no enseña: jerarquiza. No es un reclamo, pero es un índice. Una tercera función será la de insustituible labor de ordenación: hasta dejar dispuestas las interpretaciones triunfantes en la pintura ${ }^{6}$.

$\mathrm{Al}$ amparo del clima renovador iniciado, en 1954 se funda la revista de arte Goya, que dirige Camón Aznar y edita la Fundación Lázaro Galiano. Un hecho de singular importancia fue la creación, siete años después, de la Asociación Española de Críticos de Arte. Tuvo lugar en Madrid el 8 de abril de 1961 y fueron quince los miembros fundadores, siendo su Presidente José Camón Aznar, el Secretario General, José de Castro Arines y Luis Figuerola-Ferretti su Tesorero ${ }^{7}$. Aún cuando no figura ninguna mujer en su acta constitucional, pronto pasó a contar con miembros femeninos, siendo una de las pocas instituciones que abrió sus puertas a la actividad profesional de la mujer sin el menor veto discriminatorio. Bastaba para pertenecer a la misma ser presentado por dos de sus miembros, entregar documentación escrita de la labor realizada, que evaluaba una Comisión de ingresos y llevar más de dos años ejerciendo tareas de crítica en un medio de comunicación.

En la década de los sesenta la Asociación registra una intensa actividad. En 1966 tuvieron lugar en Madrid los Primeros Coloquios de la Crítica de Arte, con intervenciones de Gaya Nuño, Valeriano Bozal, Manuel Conde, Juan Eduardo Cirlot, Carlos Areán y José Camón Aznar. Dos años después y entre otras actuaciones, la Asociación protestaba por la escasa presencia de los críticos de arte en Televisión Española, al tiempo que alertaba a las autoridades sobre la necesidad de emplazar esculturas en el entorno urbano y en la de crear museos de arte al aire libre. En julio de 1968 la AECA impartía su Primer Curso de Critica de Arte en Santander, en la Universidad Internacional de Verano y sus miembros sentaban las bases de lo que sería una crítica orientadora y constructiva: una labor que preside la exigencia del mejor hacer y pasa por aproximar la obra de arte al contemplador y por establecer un diálogo con la obra y con el artista.

A finales de los sesenta ingresan en la Asociación las primeras críticas de arte. Elena Florez, Lina Font, Teresa Soubriet, Mercedes Lazo y 
Consuelo de la Gándara, son pioneras. A sus nombres se suman en los años setenta y ochenta los de María Teresa Ortega Coca, Carmen Bermúdez, Rosa Martínez de Lahidalga, Catherine Coleman, Amparo Martí, Carmen Rocamora, Julia Sáez Angulo, Gianna Prodan y un largo etcétera, en Madrid; Beatriz Guttmann y Teresa Beriguistain, en Valencia; María Luisa Borrás, María-Josep Balsach, María del Mar Arnús, Maria Teresa Blanch, Gloria Bosch, Teresa Camps, Maria José Corominas, Victoria Combalía en Barcelona. En las dos últimas décadas la presencia de la mujer en el terreno de la crítica de arte supera incluso a la del hombre y se hace extensiva a la casi totalidad de las comunidades autónomas.

Trazamos a continuación el perfil de algunas de ellas, como indicativo de la actividad que la mujer ha desempeñado y desempeña en este campo. Elena Florez ingresó en la Asociación el año 1968. En el Tercer Programa de Radio Nacional de España organizó y dirigió los Coloquios de Arte y en el diario El Alcázar ejerció la crítica entre 1968 y 1988, colaborando asiduamente en las revistas Bellas Artes y Goya, donde continúa haciendo la recesión de libros de arte. Nombrada Academica Correspondiente de la Real de BB.AA. de S. Fernando en 1996, colabora actualmente en el Boletín de la misma y hace extensiva su labor a la participación en jurados, conferencias y congresos.

Teresa Soubriet llega al mundo del arte de la mano de una seria actividad literaria y poética. Casada con el pintor y crítico de arte Ceferino Moreno, comisario en Bienales internacionales, toda su vida gira en torno al pensamiento y a la creación artística, al tiempo que ha ejercido la docencia como profesora de literatura hasta hace pocos años. La pintora y escritora Carmen Bermúdez, licenciada en Bellas Artes por la Facultad de San Carlos de Valencia y Doctora por la Complutense de Madrid, desarrolla tempranamente tareas de crítica en su Jaén natal, colaborando en el periódico Jaén. Cofundadora del grupo literario y revista El Olivo, ha publicado libros de poesía y celebrado numerosas exposiciones de pintura. Su ingreso en la Asociación Española de Críticos de Arte tuvo lugar en 1975 y en el seno de la misma desempeñó el cargo de Tesorera, siendo posteriormente Secretaria General de la Asociación Madrileña desde su Fundación.

Carmen Rocamora inicia su actividad como crítico de arte con anterioridad a 1988, año en que ingresa en la Asociación. Sus estudios en Lisboa sobre Arte Portugués; en Roma en la Dante Alighieri, donde profundiza en el arte griego y romano, configuran un perfil que incluye la autoria de libros como Lo fugaz y lo eterno en la pintura contemporánea, Los nuevos dioses del arte actual y Veinte museos de arte contemporáneo del mundo, entre otros. 
En Valencia, Maite Beriguistain compagina sus tareas como crítica, al tiempo que pertenece a la junta directiva de la Asociación Internacional de Críticos de Arte y es profesora del Departamento de Estética de la Universidad de Valencia; en Barcelona. Victoria Combalía ha publicado importantes estudios sobre arte y artistas contemporáneos, es miembro de la Fundación Rockefeller y dirige el Centro de arte Tecla Sala.

Imposible resumir el número de mujeres que a lo largo de estos años se han incorporado a las tareas de la crítica, aportando junto a una sólida formación cultural adecuada al ejercicio de la crítica, una peculiar vocación profesional. Pertenecientes a la Asociación Española de Críticos de Arte, que se halla en correspondencia con la Internacional, y con las asociaciones autonómicas, o fuera de toda vinculación institucional, son muchas las mujeres que participan en la actualidad en la marcha del arte: unas desde la reflexión y estudio del panorama artístico, en relación directa con el artista y su obra; otras como gestoras de exposiciones o directoras de museos. Sirvan de ejemplo, por citar algunas profesionales entre las más destacadas, María Corral, directora hasta 1994 del Centro de Arte Reina Sofía y que dirige en la actualidad la colección de la Fundación La Caixa; Gloria Moure, exdirectora del Centro Gallego de Arte Contemporáneo o Carmen Jiménez, exdirectora del Centro de Arte Reina Sofía, comisaria de exposiciones nacionales o internacionales de primer orden, que hoy ocupa altas instancia en el Guggenheim de Nueva York.

La crítica de arte ha visto ampliados sus horizontes profesionales dando cabida a los nuevos roles surgidos por una actividad que pasa de lo rigurosamente artístico a lo social, a lo político y lo económico, exigiendo la aparición de nuevos profesionales. No por ello deja de ser necesaria la actividad de quienes desempeñan tareas de auténtica crítica.

La labor desempeñada por la mujer en el ejercicio de la crítica de arte en el último tercio del siglo es notoria. Autora de libros, de artículos y ensayos que ven la luz en diarios y en revistas especializadas, participa en jurados nacionales e internacionales, comisaría de exposiciones y con frecuencia interviene como conferenciante. Enseñar a mirar la obra de arte es tarea primordial que mueve a su actividad, en un intento no solo de informar sino de aproximar la obra de arte al contemplador y hacer que sea cada vez más estrecha la línea divisoria entre arte y público

En diciembre de 1986, con motivo de cumplirse el XXV aniversario de la Asociación Española de Críticos de Arte y coincidiendo con la celebración del III Congreso Nacional, quien esto escribe pronunciaba una conferencia sobre $E l$ reto de la crítica de arte ante el futuro ${ }^{8}$, en el Centro Cultural Conde Duque, en Madrid. El protagonismo del arte en la sociedad contemporánea - decía entonces- exige del crítico llevar a cabo un 
análisis de la cultura y sumar a la vivencia de la obra la exigencia de ordenar el pensamiento y de ampliar el conocimiento a cuanto afecta al saber de su tiempo.

Partía de considerar al crítico como a un espectador sensible con visión de futuro; un apasionado por conocer cuanto el hombre de su tiempo sueña, realiza o crea; un informador directo de lo que la obra es y un iniciador al mundo que contiene. Y estimaba como obligación inherente al mismo la de cumplir una misión crítica orientada por el estudio y el análisis, y una misión reflexiva y filosófica que trasciende con mucho a la simple tarea de informar, de anotar, de registrar cuanto la obra es.

Ha habido momentos en que el oficio de la crítica de arte ha conocido el desprestigio. Hoy se ve salpicado por la incomprensión y por exigencias extraartísticas. La situación en que se desempeña la crítica de arte es, en sí misma, difícil. De una parte, el público exige que se le diga lo que es bueno y por qué, y pide una casi definición de claridades que el arte no admite en su totalidad y que el crítico no puede desvelar. El crítico tiene la obligación de permanecer lo más cerca posible, no solo de cuanto es arte, sino de cuanto artístico se gesta en sus proximidades. Además de ser un testigo de excepción, esta obligado a analizar el fenómeno artístico en sí mimo pero también como ingrediente determinante de la cultura moderna.

El nombre de María Zambrano, ilumina de modo excepcional el panorama del pensamiento en torno al arte y a lo artístico A través de su pensamiento nos llega una manera de conocer el arte y el hecho artístico, que se halla impregnada de poesía y de filosofía, de sentimiento, sensibilidad e inteligencia.

A lo largo de un complejo itinerario vital que pasa por diversas ciudades españolas -Madrid, Barcelona, Valencia-y por países como Chile; Cuba, donde permanece 13 años; Roma, donde residirá de 1953 a 1964; Francia, Suiza y al fin España, a donde regresa en 1984, mantuvo una estrecha relación con el mundo del arte a través del permanente contacto con pintores, escultores y artistas. De la importancia que en su vida tuvo la pintura habla en el prólogo a su libro Algunos lugares de la pintura, donde recopila artículos y ensayos ${ }^{9}$. «La pintura es una presencia constante, existe para mí, ha existido siempre, como un lugar privilegiado donde detener la mirada»...»Esa revelación, ese lugar privilegiado que se da en la pintura, no solo depende de los pintores, sino también de la predisposición de quien mira y aporta la revelación solo a determinadas miradas».

El libro ofrece páginas inolvidables sobre la luz en Zurbarán, y su hermanamiento con Grís, en un espacio vacío; observaciones penetrantes so- 
bre la a historia de España «que pasa por la luz de Zurbarán y por el no estilo de Velázquez»; o cuando revela esa primera condición de la pintura española que es contradicción: esplendor y ensimismamiento. Páginas también sobre Picasso, Miró, Tàpies, Ramón Gaya, Gregorio Prieto, Angel Alonso, Juan Soriano, Baruj Salinas, Luis Fernández y otros artistas a los que trató y de los cuales escribió con una inteligencia y profundidad digna de la mejor crítica.

A propósito de la comunicación y entendimiento que debe establecer el crítico con el artista y su obra, habla Juan Eduardo Cirlot ${ }^{10}$ de un género muy extraño, pero de los superiores, que es el de la complicidad. Una complicidad que se advierte en el plano «metafísico y espiritual».

En esta misma línea de entendimiento, en el Congreso Internacional de Críticos de Arte que acabada de celebrase en Londres, la ponencia presentada por el escultor Antony Gormley venia a destacar la importancia que en la gestación de la obra tiene él critico. En la suya, concretamente, crítico y artista habían caminado al unísono por una senda de silencios y estímulos, en la que a veces le había precedido el crítico con sus observaciones y pensamientos en torno a la obra.

La crítica de arte no carece de sentido en el momento actual pero con demasiada frecuencia se limita casi exclusivamente su papel al de informadora o constatadora de cuanto confluye en la red que teje el arte contemporáneo. La cultura que genera la vida contemporánea trata de satisfacer una demanda surgida de la realidad contemporánea que es problemática. Ante la confusión generada por la diversidad del fenómeno artístico es necesario dar impulso a la investigación que ahonde en raíces aún vivas de las que emerge ese nuevo múltiple y diverso. Hay quien cree asistir al resurgir de la gran tradición abstracta de la pintura moderna, pero no puede dejar de constatar que se produce también el resurgir de una auténtica y frecuente vocación figurativa.

$\mathrm{El}$ arte refleja a estas alturas del siglo un proceso general de disolución, un estado de crisis. Sus producciones son, a menudo, más interesantes como síntoma del clima de la vida moderna que desde el punto de vista artístico y no se ven todavía elementos constructivos, estables o duraderos. Domina el panorama un conjunto de tendencias intimistas, expresiones características de una espiritualidad que tal vez bajo el efecto de un traumatismo, se retiran al mundo de la subjetividad privada del artista.

George Steiner, escritor, pensador y crítico francés nacido el año 30 del siglo reflexionaba recientemente sobre si ha pasado el tiempo de las grandes obras o, por el contrario, nos espera un renacimiento. Aun cuando no se atreve a negar que tal vez nos hallemos en vísperas de una gran 
renovación, apunta a los científicos como los hombres que están llenos de esperanza y de confianza en sus conquistas futuras.

$\mathrm{Su}$ duda sobre que sea el arte el que inicie la marcha creadora la basa en el hecho de que, hasta hace poco, las grandes creaciones han tenido una relación directa con la cuestión de la existencia o inexistencia de Dios. Si entramos en una época en la que tal cuestión carece de sentido, se trata de una época en la que toda trascendencia ha sido expulsada violentamente. Entonces cabe preguntarnos sobre si habrá todavía obras de la misma dimensión, si tendrán el mismo alcance y la misma ambición.

Es posible que el hombre de hoy se encuentre menos mal en la atmósfera de un cierto arte muy moderno precisamente porque este refleja una especie de autodisolución del arte. Dominan en el mismo tendencias que tienen como consigna el arte puro en el sentido de puro formalismo y en el cual el contenido es insignificante. Y la corriente opuesta, en la que confluyen cuantas tendencias son reflejo del proceso general de disolución. Sus producciones son a menudo interesantes, no desde el punto de vista artístico sino como síntomas del clima de la vida moderna. Reflejan un estado de crisis, pero no aportan nada constructivo, estable o duradero.

El mercado global con su insaciable apetito por'abarcarlo todo se ha encargado de que no haya relajación en el sistema y que cualquier expresión cultural que no sea transparente al mercado sea ignorada. Asistimos a una cultura internacional de comunicación y espectáculo en la que el arte ha perdido su capacidad transformadora y en gran medida también su capacidad crítica.

Ante este panorama la crítica de arte tiene que replantearse sus deberes. Arte y crítica de arte no son dos formas ajenas de cultura sino próximas y están llamadas a complementarse. Confiamos en una crítica que, esté realizada por un hombre o una mujer, abarque la reflexión y el análisis, desde el conocimiento. Al artista y del crítico los creemos capaces de dar noción de esos corpúsculos de luz que yacen en la esencia misma del hombre y que perfilan el camino hacia un mundo mejor y posible en cuya construcción todos estamos comprometidos.

\section{Notas}

1 GaYa Nuño, J. A. (1975): Historia de la crítica de arte en España. Ibérico Europea de Ediciones, Madrid.

2 Ob. cit. pp. 307 a 312

3 SÁnchez Camargo, M. (1963): Historia de la Academia Breve de Crítica de Arte: Homenaje a genio d’Ors. Manuel Sánchez Camargo. Madrid. 


\section{La mujer, crítico de arte}

4 D’Ors, E. (1967): Mis Salones. Itinerario del Arte Moderno en España. Aguilar Madrid, p. 67.

5 Campo Alange, Condesa De. (1944): María Blanchard. Hauser y Menet. Madrid.

6 Expone estos criterios en la presentación de la Quinta Exposición Antológica de la Academia Breve, 1948-1949.

7 I CONGRESO NACIONAL DE LA ASOCIACIÓN ESPAÑOLA DE CRÍTICOS DE ARTE. ESTATUTO GUÍA (1983). Asociación Española de Críticos de Arte, Madrid, pp. 12-17.

8 MARTinez DE LAHIDALGA, R. (1986):

\section{Bibliografía}

CAmpo Alange, Condesa De (1944): María Blanchard. Hauser y Menet. Madrid.

D’ORS, E. (1967): Mis Salones. Itinerario del Arte Moderno en España. Aguilar. Madrid.

GAYA NUÑO, J. A. (1975): Historia de la crítica de arte en España. Ibérico Europea de Ediciones. Madrid

MARTINEZ DE LAHIDALGA, R. (1986): El reto de la crítica de arte ante el futuro. Conferencia pronunciada en el Centro Cultural Conde Duque de Madrid, el 8 de diciembre de 1983, en el IIII Congreso de la Asociación de Críticos de Arte

SÁnchez Camargo, M. (1963): Historia de la Academia Breve de Críticos de Arte. Homenaje a Eugenio d'Ors. Manuel Sánchez Camargo. Madrid

ZambRANo, M. (1989): Algunos lugares de la pintura. Recopilación Amalia Iglesias. Espasa Calpe. Madrid. 